\title{
Analysis of static and dynamic balance in healthy elderly practitioners of Tai Chi Chuan versus ball- room dancing
}

\author{
Miguel Antônio Rahal,' Angélica Castilho Alonso," Felix Ricardo Andrusaitis," Thuam Silva Rodrigues," \\ Danielli Souza Speciali, "IIII* Júlia Maria D'Andréa Greve, ${ }^{\text {IV }}$ Luiz Eugênio Garcez Leme ${ }^{\mathrm{V}}$
}

'Instituto de Ortopedia da Faculdade de Medicina da Universidade de São Paulo (IOTHCFMUSP), Geriatrician, São Paulo/SP, Brazil. "Instituto de Ortopedia da Faculdade de Medicina da Universidade de São Paulo (IOTHCFMUSP), Movement Studies Laboratory, São Paulo/SP, Brazil. "' Hospital Israelita Albert

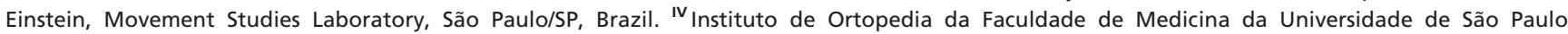

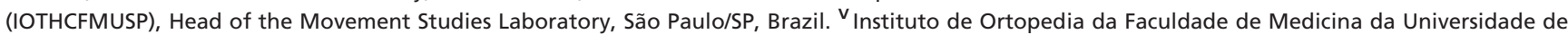
São Paulo (IOTHCFMUSP), Head of the Clinical Sector and Geriatric Group, São Paulo/SP, Brazil.

OBJECTIVE: To determine whether Tai Chi Chuan or ballroom dancing promotes better performance with respect to postural balance, gait, and postural transfer among elderly people.

METHODS: We evaluated 76 elderly individuals who were divided into two groups: the Tai Chi Chuan Group and the Dance Group. The subjects were tested using the NeuroCom Balance Master ${ }^{\circledR}$ force platform system with the following protocols: static balance tests (the Modified Clinical Tests of Sensory Interaction on Balance and Unilateral Stance) and dynamic balance tests (the Walk Across Test and Sit-to-stand Transfer Test).

RESULTS: In the Modified Clinical Test of Sensory Interaction on Balance, the Tai Chi Chuan Group presented a lower sway velocity on a firm surface with open and closed eyes, as well as on a foam surface with closed eyes. In the Modified Clinical Test of Sensory Interaction on Unilateral Stance, the Tai Chi Chuan Group presented a lower sway velocity with open eyes, whereas the Dance Group presented a lower sway velocity with closed eyes. In the Walk Across Test, the Tai Chi Chuan Group presented faster walking speeds than those of the Dance Group. In the Sit-to-stand Transfer Test, the Tai Chi Chuan Group presented shorter transfer times from the sitting to the standing position, with less sway in the final standing position.

CONCLUSION: The elderly individuals who practiced Tai Chi Chuan had better bilateral balance with eyes open on both types of surfaces compared with the Dance Group. The Dance Group had better unilateral postural balance with eyes closed. The Tai Chi Chuan Group had faster walking speeds, shorter transfer times, and better postural balance in the final standing position during the Sit-to-stand Test.

KEYWORDS: Postural Balance; Aging; Motor Control; Fall Prevention.

Rahal MA, Alonso AC, Andrusaitis FR, Rodrigues TS, Speciali DS, D'Andréa Greve JM, et al. Analysis of static and dynamic balance in healthy elderly practitioners of Tai Chi Chuan versus ballroom dancing. Clinics. 2015;70(3):157-161.

Received for publication on August 4, 2014; First review completed on September 10, 2014; Accepted for publication on December 8, 2014

E-mail: danispeciali@ig.com.br

*corresponding author

\section{INTRODUCTION}

The aging process and the chronic diseases that affect older adults lead to balance disorders, which makes these individuals more susceptible to falls. The consequences of a fall, even one without physical injury, are grave: falls can lead to a fear of falling, poor quality of life, loss of independence, and nursing home admission. Thus, it is

Copyright (C) 2015 CLINICS - This is an Open Access article distributed under the terms of the Creative Commons Attribution Non-Commercial License (http:// creativecommons.org/licenses/by-nc/3.0/) which permits unrestricted noncommercial use, distribution, and reproduction in any medium, provided the original work is properly cited.

No potential conflict of interest was reported.

DOI: $10.6061 /$ clinics/2015(03)01 necessary to investigate procedures to reduce the incidence of falls in this population $(1,2)$.

Balance is important for maintaining postural equilibrium and thereby avoiding falls. Aging may affect the central nervous system (i.e., changes in brain volume) and neuromuscular system properties (i.e., loss of sensory and motor neurons), leading to deficits in balance and gait performance (3). According to Shumway-Cook and Woollacott (4), balance can be subdivided into static/ dynamic steady-state (i.e., maintaining a steady position while sitting, standing and walking), proactive (i.e., anticipating a predicted disturbance), and reactive (i.e., compensation for a disturbance) balance.

Maintaining balance requires integrated actions of the visual, vestibular and nervous systems to process sensory information and send appropriate motor responses continuously (2). 
Walking is a complex phenomenon that requires repetitive and coordinated movements of the limbs to effectively and safely move the body. Biological aging causes structural changes and functional capacity losses, with direct decreases in balance and walking (1-5).

Several interventions should be implemented to prevent falls, including specific exercises to improve balance and walking, to treat postural hypotension, to adapt to the physical environment, to treat cardiovascular disorders, and to mediate drug interactions (1).

The number of elderly people who practice some type of physical activity has been exponentially growing in recent years, and there is a push to find the best indications for postural balance and functional activities (5).

In recent years, the practice of Tai Chi Chuan has been linked to improved postural balance, cardiorespiratory parameters, muscle strength, flexibility and memory $(3,4)$. Currently, there is a consensus that Tai Chi Chuan is an exercise technique that promotes the improvement of postural balance (5). It involves slow, gentle movements performed with body awareness, attention to breathing, and meditation. A meta-analysis conducted by Wu (5) demonstrated that to prevent falls, exercises are needed that both challenge the balance and are maintained for long periods of time, both of which are specific characteristics of Tai Chi Chuan. In a controlled study, Nguyen and Kruse (6) observed a reduction in the number of falls among the Tai Chi Chuan Group, and Leung et al. (7) recommended Tai Chi Chuan in a systematic review as an alternative treatment for improving balance and preventing falls.

Alternatively, ballroom dancing involves fast and frenetic movements, turns and changes of direction and has very distinct characteristics from Tai Chi Chuan, demanding the use of different sensory and motor systems. This activity combines emotions, social interaction, sensory stimulation, motor coordination and musicality (8). Kattenstroth (9) demonstrated that a group of individuals who danced semiprofessionally showed higher performance in cognition, reaction time, postural balance and motor and tactile performance compared with a group of amateur dancers.

Borges et al. evaluated the effect of a ballroom dancing program on institutionalized elderly people and observed an improvement in functional independence and postural balance in these individuals (10).

Interventions in the elderly require care with the specificity of the task performed and with the functional evaluation, which must be performed with reliable and reproducible instruments $(11,12)$. Certain questions remain unanswered in the literature, including whether dynamic activities, such as ballroom dancing, provide the specificity demanded by postural balance compared to the general consensus in the present literature.

Therefore, the aim of this study was to determine whether Tai Chi Chuan or Ballroom Dancing promotes better postural balance, gait and postural transfer among active and independent elderly people using static and dynamic posturography parameters.

\section{METHODS}

This is an observational cross-sectional study that was carried out without intervention. All of the individuals were analyzed at the Movement Laboratory of the Institute of Orthopedics and Traumatology, Hospital das Clínicas,
Faculdade de Medicinada Universidade de São Paulo (LEM-IOT-HCFMUSP). Written informed consent was required for participation in the study. The study was performed after securing the approval of the institutional Ethics Committee (no. 401/06).

\section{Subjects}

A total of 76 healthy elderly individuals were evaluated, all of whom engaged in physical activity three times per week for 45 to 60 minutes, burning approximately $2000 \mathrm{kcal}$ per week. They were divided into two groups: a Tai Chi Chuan Group (TCCG) and a Dance Group (DG). The characteristics of the sample are shown in Tables 1 and 2.

The volunteers were selected by a geriatrician by detailed anamnesis.

\section{Inclusion criteria}

- Both groups: aged 60 years or older; walking independently without the use of auxiliary aids; able to climb eight steps, independence in daily living activities; able to move a horizontal leg-press once with each leg at a load equal to the body mass (kg); practicing physical activity, i.e., burning approximately two thousand kilocalories (Kcal) per week; and an absence of falls during the last three months, neurological changes, musculoskeletal changes, surgical interventions of the lower limbs and dementia.

- Dance Group: practicing ballroom dancing at least three times per week for at least one year.

- Tai Chi Chuan Group: practicing the technique of Tai Chi Chuan exercises (Lian-Gong Modality) at least three times per week for at least one year.

\section{Measurements}

First, the volunteers were instructed verbally and through a video explaining how to perform the tests. After demonstrating their understanding of the task to be performed, the subjects were positioned and instructed to start the test when the word "go" appeared on the screen. All of the tests had a duration of 10 seconds and were repeated three times. All tests were performed in the afternoon on the same day.

The subjects were tested using the NeuroCom Balance Master ${ }^{\circledR}$ force platform system (NeuroCom International, Inc., Clackamas, OR, USA), which includes a computer linked to a force plate that records data with the aid of piezoelectric crystal transducers. The force-plate data included the $X( \pm 0.08 \mathrm{~cm})$ and $Y( \pm 0.25 \mathrm{~cm})$ positions of the center of vertical force and the total vertical force $( \pm 0.1 \mathrm{~N})$ at a sampling rate of $100 \mathrm{~Hz}$. With this system, the transducers transmit pressure every $10 \mathrm{~ms}$ to a computer;

Table 1 - Characterization of the sample by gender and group.

\begin{tabular}{lcccccc}
\hline \multirow{5}{*}{ Group } & \multicolumn{9}{c}{ Gender } & & \\
\cline { 2 - 5 } & Female & $\%$ & Male & $\%$ & \multirow{2}{*}{ Total } & $\%$ \\
\hline Tai Chi Chuan & 39 & 76.5 & 12 & 23.529 & 51 & 67.1 \\
Dance & 17 & 68.0 & 8 & 32.0 & 25 & 32.8947 \\
Total & 56 & 73.6842 & 20 & 26.3 & 76 & 100.0 \\
& $\chi^{2}=0.621$ & $p=0.431$ & & \\
\hline
\end{tabular}


Table 2 - Characterization of the age of the sample: mean, median, maximum and minimum values.

\begin{tabular}{lcc}
\hline Age & Dance Group & Tai Chi Chuan Group \\
\hline Mean & 70.3 & 76.8 \\
Median & 69.1 & 77.5 \\
Maximum & 85 & 88 \\
Minimum & 60 & 67 \\
$\mathbf{N}$ & 25 & 51 \\
$\mathbf{z = 4 . 2 1 8 ^ { * }}$ & & $\mathrm{P}<0.001$ \\
\hline
\end{tabular}

then, the dynamic center of gravity of the subject is calculated, and the sway velocity over a defined period is obtained. The equipment has good reliability and reproducibility between researchers (ICC 0.53 to 0.81$)(12,13)$. The NeuroCom Balance Master ${ }^{\circledR}$ has multiple testing protocols designed to examine balance. This study used the Modified Clinical Test of Sensory Interaction on Balance (mCTSIB) and Unilateral Stance for the static balance protocol and the Walk Across Test and Sit-to-stand Transfer Test for the dynamic balance protocol $(14,15)$.

\section{Static balance protocols}

Modified Clinical Test of Sensory Interaction on Balance (mCTSIB): This test quantifies the sway velocity $(\%)$ with the subject standing under four test conditions: 1) on a firm surface with eyes open, 2) on a firm surface with eyes closed, 3) on a foam surface with eyes open and 4) on a foam surface with eyes closed. Unilateral Stance: This test quantifies the sway velocity $(\%)$ with the subject standing on one leg under four conditions: 1) right leg with open eyes, 2) right leg with closed eyes, 3) left leg with open eyes and 4) left leg with closed eyes.

\section{Dynamic balance protocols}

Walk Across Test: This test quantifies the speed of progression, step width and final postural sway. Sit-tostand Transfer Test: The parameters measured were the mean weight transfer and the center of gravity (COG) sway velocity while standing up.

\section{Statistical analysis}

Data analysis was performed using SPSS version 17.0 for Windows. Statistical significance was set at $\mathrm{p}<0.05$. For the association between genders in the Tai Chi Chuan and Dance Groups, the chi-square test $\left(\chi^{2}\right)$ was used. The MannWhitney $\mathrm{U}$ test was used to compare ages and the static and dynamic protocol test results between groups.

\section{RESULTS}

In the mCTSIB, the Tai Chi Chuan Group presented a lower sway velocity $(\%$ s) on a firm surface with open and closed eyes and on a foam surface with closed eyes.

In the Unilateral Stance test, the Tai Chi Chuan Group presented a lower sway velocity $(\%$ ) with open eyes (right and left), whereas the Dance Group presented a lower sway velocity $(\%$ s) with closed eyes.

In the Walk Across Test, the Tai Chi Chuan Group presented a faster walking speed than that of the Dance Group.

In the Sit-to-stand Test, the Tai Chi Chuan Group presented a shorter transfer time from the sitting to standing position with less sway in the final standing position (Table 3).

\section{DISCUSSION}

The elderly individuals who practiced Tai Chi Chuan had better static bilateral postural balance with open or closed eyes compared with the Dance Group on both types of support used. The Dance Group had better unilateral postural balance with closed eyes. The Tai Chi Chuan Group had faster walking speeds, shorter transfer times, and better postural balance in the final standing position in the Sit-to-stand Test.

Table 3 - Comparison between the Tai Chi Chuan Group and Dance Group.

\begin{tabular}{|c|c|c|c|c|}
\hline & & Tai Chi Chuan Group Median & Dance Group Median & $P$ \\
\hline \multicolumn{5}{|l|}{ mCTSIB test ( $\%$ s) } \\
\hline \multirow[t]{2}{*}{ Open Eyes } & Firm Surface & 0.200 & 0.400 & $<0.001 *$ \\
\hline & Foam Surface & 0.800 & 0.300 & 0.172 \\
\hline \multirow[t]{2}{*}{ Closed Eyes } & Firm Surface & 0.200 & 0.900 & $0.029 *$ \\
\hline & Foam Surface & 1.400 & 1.950 & $0.002^{*}$ \\
\hline \multicolumn{5}{|l|}{ Unilateral Stance ( $\%$ s) } \\
\hline \multirow[t]{2}{*}{ Open Eyes } & Right Leg Stance & 1.000 & 1.300 & $<0.001^{*}$ \\
\hline & Left Leg Stance & 1.100 & 1.200 & $0.002 *$ \\
\hline \multirow[t]{2}{*}{ Closed Eyes } & Right Leg Stance & 3.200 & 1.900 & $<0.001 *$ \\
\hline & Left Leg Stance & 3.500 & 2.200 & $0.009 *$ \\
\hline \multicolumn{5}{|l|}{ Walk Across Test } \\
\hline Step width $(\mathrm{cm})$ & & 11.000 & 10.300 & 0.800 \\
\hline Speed $(\mathrm{cm} / \mathrm{s})$ & & 23.600 & 18.200 & $0.008^{*}$ \\
\hline End sway velocity ( $/ \mathrm{s})$ & & 4.600 & 6.300 & 0.107 \\
\hline \multicolumn{5}{|l|}{ Sit-to-stand } \\
\hline Weight Transfer (sec) & & 0.280 & 0.340 & $0.0036 *$ \\
\hline COG Sway Velocity ( $\%$ s) & & 4.000 & 5.200 & $0.045^{*}$ \\
\hline
\end{tabular}

Test: Mann-Whitney U test.

${ }^{*} \mathrm{p}<0.05$.

Legend: $\mathrm{mCTSIB}=$ Modified Clinical Test of Sensory Interaction on Balance; $\mathrm{COG}=$ center of gravity; $\% / \mathrm{s}=$ degrees/second. 
In the mCTSIB test, which analyzes posture in relation to visual and proprioceptive information in either an isolated or associated form, the Tai Chi Chuan Group presented a lower sway velocity with bilateral standing on both stable and unstable surfaces with closed eyes and on a stable surface with open eyes, demonstrating better balance than that of the Dance Group.

These results can be explained by the technique underlying the Tai Chi Chuan exercises, which comprise a series of slow movements that are combined to form a continuous sequence so that the body is constantly transitioning from standing on both legs to standing on one leg, emphasizing shifts and changes to the support base $(16,17)$ Mao et al. (18) evaluated the characteristics of foot movement during Tai Chi Chuan exercises and during walking and showed that individuals who practice this technique had better doublelimb support. Slow, concentrated training movements can create slower motor patterns, which help to improve postural balance; this pattern differs from that of ballroom dancing, which comprises a series of body movements performed at a fast pace.

In the bilateral standing test with open eyes, the Tai Chi Chuan Group swayed less than the other group only on the firm surface, a finding that may represent better body adjustment mechanisms to maintain balance. Ramachandran et al. (19) demonstrated that individuals who practiced Tai Chi Chuan spent more time standing on one leg when stepping over an obstacle and that the elements of meditation, body awareness, long periods of movement, weight transfer and base narrowing, which are all components of the technique, could explain these results. Wolf et al. (20) found no differences in unilateral ability to balance between a group trained in Tai Chi Chuan and a group trained on a computerized platform, but the Tai Chi Chuan Group had less fear of falling. The results are consistent with those of the present study, which shows that the practice of Tai Chi Chuan helps improve static postural balance. However, when standing on one leg with closed eyes, the Dance Group showed better results; this finding is similar to the findings reported by Kattenstroth et al. (9), who showed that a group of competitive dancers showed better performance on the Romberg Test with closed eyes than did a group of amateur dancers.

The Tai Chi Chuan Group performed the Sit-to-stand Transfer Test more quickly and with less sway in the final standing position; these results were similar to those of Tsang \& Huy-Chan (21), who reported that people who practiced Tai Chi Chuan showed faster speed and greater movement control in the stability limit test. Fong and $\mathrm{Ng}$ (22) used clinical functional range tests and found that individuals who practiced Tai Chi Chuan showed a wider range of movements and a reduced loss of balance. In the rhythmic weight protocol of the same equipment used in this study, Lin et al (16) demonstrated that the Tai Chi Chuan Group performed better with respect to the rate of anteroposterior weight discharge and directional control. Fong and $\mathrm{Ng}$ (22) demonstrated that compared with a group of beginners, a group who trained for more than a year showed faster reaction times and hamstring and calf muscle reflexes and the ability to maintain balance for longer periods of time.

The Tai Chi Chuan Group had faster walking speeds, which meant that anticipatory and/or compensatory adjustments were better in this group. The recovery of balance at the end of the Sit-to-Stand Transfer Test was also faster, although this difference was not statistically significant. This observation was in agreement with $\mathrm{Wu}$ (21), who analyzed the biomechanical characteristics of stepping among elderly practitioners of Tai Chi Chuan, showing that the time spent in preparation to start walking and the time of contact between the foot and the ground were reduced and the stride length was increased compared with those of the control group.

The clinical walking tests performed by Fong and $\mathrm{Ng}(22)$ and Taylor et al. (24) showed an increase in the walking speed and stride length in individuals who practiced Tai Chi Chuan. We did not find any data in the literature on dance for comparison.

We can affirm that Tai Chi Chuan is a physical activity that reduces postural sway and consequently reduces falls. Similar conclusions were also reported by Taylor et al. (24), who indicated a 58\% reduction in falls after a controlled intervention. The specificity of Tai Chi Chuan training (variation of support and changes of direction), particularly among the elderly, is a determining factor in improving balance and thereby preventing falls.

There are few studies on dance and postural balance. Therefore, new studies on ballroom dancing are needed to evaluate the real effects of ballroom dancing on postural balance.

Our results indicate that the practice of Tai Chi Chuan promotes an improvement in static and dynamic balance by enhancing compensatory adjustments.

\section{AUTHOR CONTRIBUTIONS}

Rahal MA and Alonso AC substantial contributions to the conception and design of the study; the acquisition, analysis and interpretation of the data; and the drafting and critical revision of the article for important intellectual content. Andrusaitis FR, Rodrigues TS, and Speciali DS substantial contributions to the conception and design of the study and the acquisition of data. D'Andréa and Greve JM substantial contributions to the conception and design of the study and final approval of the version to be published. Leme LEG substantial contributions to the conception and design of the study, final approval of the version to be published, and critical revision of the manuscript for important intellectual content.

\section{- REFERENCES}

1. Tinetti ME. Preventing falls in elderly persons. N Engl J Med. 2003; 348(1):42-9.

2. Li JX, Hong Y, Chan KM. Tai chi: Physiological characteristics and beneficial effects on health. Brit J Sport Med. 2001;35(3):148-56.

3. Granacher U, Muehlbauer T, Gruber M. A qualitative review of balance and strength performance in healthy older adults: impact for testing and training. J Aging Res. 2012;2012:708905.

4. Shumway-Cook A, Woollacott MH: Motor control: translating research into clinical practice. 3rd edition. Philadelphia, PA: Lippincott Williams \& Wilkins; 2007.

5. Wu G. Evaluation of the effectiveness of Tai Chi for improving balance and preventing falls in the older population-A review. J Am Geriatr Soc. 2002;50(4):746-54, http://dx.doi.org/10.1046/j.1532-5415.2002.50173.x.

6. Nguyen $\mathrm{MH}$, Kruse A. A randomized controlled trial of Tai chi for balance, sleep quality and cognitive performance in elderly Vietnamese. Clin Interv Aging. 2012;7:185-90, http:/ / dx.doi.org/10.2147/CIA.S32600.

7. Leung DP, Chan CK, Tsang HW, Tsang WW, Jones AY. Tai chi as an intervention to improve balance and reduce falls in older adults: A systematic and meta-analytical review. Altern Ther Health Med. 2011; 17(1):40-8.

8. Kattenstroth JA, Kalisch T, Kolankowska I, Dinse HR. Balance, sensorimotor, and cognitive performance in long-year expert senior ballroom dancers. J Aging Res. 2011:1-10.

9. Kattenstroth JA, Kolankowska I, Kalisch T, Dinse HR. Superior sensory, motor, and cognitive performance in elderly individuals with multi-year dancing activities. Front Aging Neurosci. 2010;2 pii:31. 
CLINICS 2015;70(3):157-161

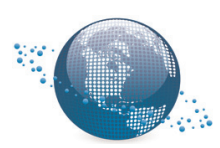

Postural balance: Tai Chi versus ballroom dancing Rahal MA et al.

10. Borges EGS, Coder SA, Vale RG, Cruz TH, Carvalho MC, Pinto FM, et al. The effect of ballroom dance on balance and functional autonomy among the isolated elderly. Arch Gerontol Geriatr. 2012;55(2):492-6, http://dx. doi.org/10.1016/j.archger.2011.09.004.

11. Ainsworth BE, Haskell WL, Leon AS, Jacobs DR Jr, Montoye HJ, Sallie JF, et al. Compendium of Physical Activities: Classification of energy costs of human Physical activities. Med Sci Sports Exerc. 1993;25(1):71-80, http:/ /dx.doi.org/10.1249/00005768-199301000-00011.

12. Alonso AC, Luna NMS, Mochizuki L, Barbieri F, Santos S, Grove JM. The influence of anthropometric factors on postural balance: the relationship between body composition and posturographic measurements in young adults. Clinics. 2012;67(12):1433-41, http://dx.doi.org/10.6061/clinics/ 2012(12)14.

13. Loughran S, Pennant N, Kishore A, Swan IR. Interobserver reliability in evaluating postural stability between clinicians and posturography. Chin Otolaryngol. 2005;30(3):255-7, http:/ /dx.doi.org/10.1111/j.1365-2273.2005. 00988.x.

14. Tomomitsu MSV, Alonso AC, Morimoto E, Bobbin TG, Grove JM. Static and dynamic postural control in low-vision and normal-vision adults. Clinics. 2013;68(4):517-21, http:/ /dx.doi.org/10.6061/clinics/2013(04)13.

15. Rahal MA, Andrusaitis FR, Rodrigues TS, Alonso AC, Gree JMD, Lee LEG. Gait, posture and transfer assessment among elderly practitioners and non-practitioners of Tai Chi Chuan. Health. 2013;(5):117-21, http:/ / dx.doi.org/10.4236/health.2013.512A016.

16. Lin YC, Wong AM, Chou SW, Tang FT, Wong PY. The effects of Tai Chi Chian on postural stability in the elderly: preliminary report. Chang Sung Med J. 2000;23(4):197-204.
17. Hes CJ, Gregor RJ, Weddell DE, Oliver A, Smith DW, Fleming RP, et al. The influence of Tai Chi training on the center of pressure trajectory during gait initiation in older adults. Arch Phys Med Rehabil. 2004;85(10):1593-8, http://dx.doi.org/10.1016/j.apmr.2004.01.020.

18. Mao DW, Hong Y, Li JX. Characteristics of foot movement in Tai Chi exercise. Phys Then. 2006;86(2):215-22.

19. Ramachandra AK, Rosengren KS, Yang Y, Hsiao-Wecksler ET. Effect of Tai Chi on gait and obstacle crossing behaviors in middle-aged adults. Gait Posture. 2007;26(2):248-55, http://dx.doi.org/10.1016/j.gaitpost. 2006.09.005.

20. Wolf SL, Barnhart HX, Ellison GL. The effect of Tai Chi Qua and computerized balance training on postural stability in older subjects. Phys Then. 1997;77(4):371-81.

21. Tang WWN and C. Huy-Chan WY. Effects of tai chi on joint proprioception and stability limits in elderly subjects. Med Sci Sports Exerc. 2003;35(12):1962-71, http://dx.doi.org/10.1249/01.MSS.0000099110. 17311.A2.

22. Fond SM, Mg GY. The effects on sensorimotor performance and balance with Tai Chi training. Arch Phys Med Rehabil. 2006;87(1):82-7, http:// dx.doi.org/10.1016/j.apmr.2005.09.017.

23. Wu G. Biomechanical characteristics of stepping in older Tai Chi practitioners. Gait Posture. 2012;36(3):361-6, http:/ /dx.doi.org/10.1016/ j.gaitpost.2012.03.029.

24. Taylor D, Hale L, Schluter P, Waters DL, Dins EE, McCracken, et al. H Effectiveness of tai chi as a community-based falls prevention intervention: a randomized controlled trial. J Am Geriatr Soc. 2012;60(5):841-8, http://dx.doi.org/10.1111/j.1532-5415.2012.03928.x.

161 